\title{
Integrating psychometric indicators in latent class choice models
}

\author{
Ricardo Hurtubia * \\ My Hang Nguyen ${ }^{\dagger}$ \\ Michel Bierlaire \\ 22nd November 2013 \\ Report TRANSP-OR 130423 \\ Transport and Mobility Laboratory \\ Ecole Polytechnique Fédérale de Lausanne \\ transp-or.epfl.ch
}

Aurélie Glerum

\begin{abstract}
Latent class models are a convenient and intuitive way to introduce taste heterogeneity in discrete choice models by relating attributes of the decision makers with unobserved behavioral classes, hence allowing for a more accurate market segmentation. Estimation and specification of latent class models can be improved with the use of psychometric indicators that measure the effect of unobserved attributes in the individual preferences. This paper proposes a method to introduce these additional indicators in the specification of integrated latent class and discrete choice models, through the definition of measurement equations that relate the indicators to attributes of the decision maker. The method is implemented for two modechoice case studies and compared with alternative methods to introduce indicators. Results show that the proposed method generates significantly different estimates for the class and choice models and provide additional insight into the behavior of each class.
\end{abstract}

Keywords: latent class, discrete choice, psychometrics, behavior, mode choice

\footnotetext{
*UNIVERSIDAD DE CHILE, Facultad de Arquitectura y Urbanismo, rhurtubia@ fau.uchile.cl (corresponding author)

†CITEC Ingénieurs Conseils SA, Genève, myhang.nguyen@ citec.ch

\#ÉCOLE POLYTECHNIQUE FÉDÉRALE DE LAUSANNE (EPFL), School of Architecture, Civil and Environmental Engineering (ENAC), Transport and Mobility Laboratory (TRANSP-OR), \{aurelie.glerum, michel.bierlaire\}@epfl.ch
} 


\section{Introduction}

Traditionally, travel demand models have considered quantitative variables, like travel time, cost and decision maker socioeconomics, as the principal variables that explain mode choice (BenAkiva and Lerman, 1985). However, there are more complex, unobserved factors that may have a relevant effect in the way choices in general are made. Some of these latent factors are the decision maker's lifestyle, personal attitudes or perceptions (McFadden, 1986), which can be integrated into choice models. We address that aspect in the present research.

The introduction of latent factors into discrete choice models has been treated under two main approaches: latent variable models (LVM) and latent class models (LCM). The latent variable approach deals with the explicit modeling of unobserved psychological characteristics of the decision maker, such as attitudes and perceptions. The latent class approach assumes that the population can be probabilistically segmented into discrete groups that have different preferences or perceptions and, therefore, have different choice behaviors.

Psychometric indicators are additional information that can be used to specify and estimate latent constructs. They usually reflect the preferences of decision makers on topics that are (closely or not so) related to the choice that is being analyzed/modeled. Examples of psychometric indicators range from the answers to questions about the level of agreement with a statement or the "grade" that is given to the quality of a service or object (Likert, 1932), to the set of adjectives that individuals use to characterize the topic in question (Glerum and Bierlaire, 2012).

Although the use of indicators should clearly help to estimate better latent class models, its use has been mostly developed and applied in the latent variable approach (Hess et al., 2011). However, the LCM approach has characteristics that make it, in some cases, preferable over other methods to capture heterogeneity (Greene and Hensher, 2003; Shen, 2009), like a more intuitive market segmentation that, if possible, should be improved with the integration of psychometric data.

This paper proposes a method to introduce psychometric indicators in the specification of discrete choice models with latent classes. The method uses ordinal logit models as measurement relationships between the observed answers and the "utility" a respondent of a particular class will perceive for providing each of these answers. The novel feature of this method consists of specifying the measurement relationships as class-specific structural relations between the aforementioned utility and the attributes of the decision maker/respondent. The structure of the proposed model is inspired by the Generalized Random Utility Model (Walker and Ben-Akiva, 2002). The method 
is applied on two datasets for transport mode choice but should be easily implemented in other choice contexts.

The paper is organized as follows. Section 2 reviews the use of latent class models in discrete choice models and the importance of psychometric indicators to characterize the classes. Section 3 presents the modeling approach adopted in this research and designed to provide a better specification of such models. Section 4 presents a first application of the methodology on a transportation mode choice case study conducted in the Nice area (France). Section 5 provides a second application of the methodology on a mode choice case study in the low-density areas of Switzerland. Section 6 concludes on the advantages of the proposed modeling approach.

\section{Latent class models in discrete choice analysis}

Widely used in social sciences for quantitative analysis (Lazarsfeld and Henry, 1968), latent class models were not proposed in the form of choice models with class-membership probabilities until the work of Kamakura and Russell (1989). Class-membership models explain the probability of an individual belonging to a consumer segment as a function of the consumer's characteristics; they are a powerful instrument because they allow to relate attributes of the decision maker with unobserved behavioral classes and, therefore, simplify the market segmentation process.

There is evidence in the literature suggesting that latent class models are a very convenient, flexible and intuitive way to introduce taste heterogeneity in discrete choice models. For example, Bhat (1997) applied the latent class approach to the transport mode choice problem finding that the endogenous segmentation into classes allows for better data fit and more intuitive results compared to other approaches used to capture heterogeneity. Greene and Hensher (2003), Shen (2009) and Hess et al. (2011) analyzed the LCM approach, comparing it with alternative methodologies like the Mixed Logit Model (McFadden and Train, 2000) concluding that, while both offer a good way to capture unobserved heterogeneity, experimental results suggest that the latent class approach allows for a better behavioral interpretation of the results. Keane and Wasi (2012) compared the latent class approach with several other models that account for taste heterogeneity, identifying it as the one allowing the most intuitive understanding of the patterns of heterogeneity.

Several application of integrated choice and latent class models can be found in the transport and land use-related literature. For example, the aforementioned works by Bhat (1997) and Shen (2009), applied the LCM approach to the choice of transport mode while Greene and Hensher (2003) did it for route choice. In the area of land use, Walker and Li (2007) identified different 
lifestyle classes among the population of a city, showing that the segments are key determinants in the choice of residential location. Zhang et al. (2009) used a latent class structure to model different intra-household choice mechanisms regarding car ownership. Wen and Lai (2010) used the latent class approach in the airline choice problem, identifying significantly different willingness to pay across consumer segments. Similar results were obtained by Wen et al. (2012) but in the context of the choice of mode to access stations of a high-speed train. Koutsopoulos and Farah (2012) used latent classes to identify and model different patterns (or regimes) of driving behavior for a microscopic traffic simulator.

\subsection{Psychometric indicators}

Psychometric indicators can be used improve the specification and estimation process of latent constructs (like classes) because they are a measurable manifestation of the effect of unobserved attributes in the preferences of individuals (Walker and Ben-Akiva, 2002). Moreover, the use of indicators in discrete choice models may help to identify latent classes that are not captured or described by the choice data alone (Ben-Akiva, McFadden, Train, Walker, Bhat, Bierlaire, Bolduc, Börsch-Supan, Brownstone, Bunch et al., 2002). Despite this, most methodological developments are focused on the use of indicators to estimate choice models using a LVM approach (Ben-Akiva, Walker, Bernardino, Gopinath, Morikawa and Polydoropoulou, 2002), with few examples applied under the LCM approach.

Ben-Akiva and Boccara (1995) introduced the use of indicators to the estimation of models with a latent choice set by measuring the user's perceived availability of an alternative and modeling a linear relationship between this indicator, the modeled availability and the "desirability" (a proxy of the utility) of each alternative. They find that using this approach generates better predictive results than a standard logit model and that the use of indicators allows for more robust estimates. Gopinath (1995) postulated the existence of two classes of shippers in the maritime freight choice context and used indicators to measure the latent attitude of the shippers towards different freight services attribute. Hosoda (1999) estimated mode choice models for shopping trips with latent classes that are functions of continuous latent variables like the "level of consciousness" of the traveler. In Hess et al. (2013), a continuous latent variable accounting for environmental attitudes is used as an explanatory factor of a latent class model, in the context of rail travel in the UK. In these last three cases, indicators are indirectly related to the class-membership model because they are first used to measure attitudinal latent variables which are then used as explanatory variables in class-membership models. 
In the context of tourism destination choice, Boxall and Adamowicz (2002) modeled natural park choice in Central Canada and used psychometric indicators related to motivations for taking a trip, identifying four groups of travelers. However, the group membership is a direct function of how the decision-makers respond to the questions and the model could not be used for predicting demand. Morey et al. (2006) developed a fishing-location choice model based on three classes of fishermen that were identified using attitudinal data about the characteristics of a particular fishing location. However, the response probabilities to the psychometric indicators are estimated as single, class-specific parameters and are not structurally related to attributes of the decision makers. A similar approach is proposed by Collins and Lanza (2010) in the context of social and health sciences and by Atasoy et al. (2013) in the context of transport mode choice.

In the surveyed literature, the class-membership probabilities are not directly related to indicators through measurement relationships that take into account the attributes of the decision makers. This paper proposes a method to do so, through the use of ordinal models. The specification of the class-specific measurement relationships leads to a better characterization of the classes since it integrate psychometric information. Moreover it allows to interpret the responses to psychometric indicators behaviorally.

\section{Methodology}

In this section we first present the general framework of latent class models. In a second stage, we introduce the use of psychometric indicators to help identify the classes.

\subsection{Latent class model}

Latent class models assume that discrete segments of the population have different choice behaviors, explained by different perceptions of the attributes of the alternatives, different taste parameters or different decision protocols. These differences can often be linked to the lifestyle, attitudes and even political or ideological views of the decision maker. In the context of discrete choice analysis, this translates into a class-specific utility function of choosing alternative $i$ by decision maker $n$ :

$$
U_{i n}^{s}=V^{s}\left(X_{i n}, Z_{n}, \beta^{s}\right)+\varepsilon_{i n}^{s}
$$

where $V^{s}\left(X_{i n}, Z_{n}, \beta^{s}\right)$ is the (class-specific) deterministic part of the utility function, $X_{\text {in }}$ is a vector of attributes of alternative $i, Z_{n}$ is a vector of characteristics of individual $n$ and $\beta^{s}$ is a vector 
of parameters (to be estimated) that is specific to class $s$. The term $\varepsilon_{i n}^{s}$ is a random component accounting for unobserved attributes and characteristics. Assuming an i.i.d. Extreme Value distribution for the random component, we can write the probability of an individual $n$ choosing alternative $i$, conditional on the class $s$ to which he belongs, as a logit:

$$
P_{n}(i \mid s)=\frac{e^{V^{s}\left(X_{i n}, Z_{n}, \beta^{s}\right)}}{\sum_{j \in C_{s}} e^{V^{s}\left(X_{j n}, Z_{n}, \beta^{s}\right)}}
$$

where $C_{s}$ is the set of alternatives considered by individuals belonging to class $s$. For identification purposes, we fix the scale parameter of equation (2) to 1 , this means that the absolute values of the parameters cannot be interpreted, with only their signs and statistical significance being relevant. Since classes are unobserved, it is not possible to deterministically assign an individual to a class. It is possible however to assume that the membership to a class depends on the characteristics of the decision maker, and that this relation is described by a class-membership functionf, such that:

$$
F_{n s}=f\left(Z_{n}, \gamma^{s}\right)+\xi_{n s},
$$

where $F_{n s}$ is a latent continuous variable that is related to the probability of belonging to class $s$ and can be perceived as the "utility" to belong to one class, and $\gamma^{s}$ is a vector of parameters to be estimated. Assuming that $\xi_{n s}$ are i.i.d. $E V(0,1)$, the probability for an individual $n$ to belong to a particular class $s$ is given by:

$$
P_{n}(s)=\frac{e^{f\left(Z_{n}, \gamma^{s}\right)}}{\sum_{r \in S} e^{f\left(Z_{n}, \gamma^{r}\right)}}
$$

where $S$ is the set of classes. As with equation (2), the scale parameter of equation (4) is also fixed to 1 .

Following (2) and (4) the complete probability of individual $n$ choosing an alternative $i$ is:

$$
P_{n}(i)=\sum_{s \in S} P_{n}(i \mid s) P_{n}(s) .
$$

\subsection{Latent class model with psychometric indicators}

Psychometric indicators can be introduced by assuming that the probability of giving an agreement level $I_{k}$ to the $k$ th question/indicator, with $k=1, \ldots, K$ will depend on the class of the respondent. This allows to write the joint probability of choosing $i$ and answering $I_{k}$ for individual $n$ as: 


$$
P_{n}\left(i, I_{k}\right)=\sum_{s \in S} P_{n}(i \mid s) P_{n}(s) \prod_{k=1}^{K} P_{n}\left(I_{k} \mid s\right)
$$

where $P_{n}\left(I_{k} \mid s\right)$ is the probability of answering $I_{k}$ to the $k$ th indicator if the respondent $n$ belongs to class $s$. As mentioned in Section 2.1, this probability is usually estimated directly as a single parameter or a constant.

We propose to model the response probability $P_{n}\left(I_{k} \mid s\right)$ as a function of the attributes of the respondent (or decision maker), conditional on the class. For this we consider a continuous latent construct that varies with both the characteristics and the class of the respondent, and we derive an ordered logit model from it. Our hypothesis is that, by doing so, we enhance the characterization of the class-membership model.

We focus on the case where indicators take the form of questions where an ordered response is provided. A typical example of this is when the respondent is asked about his level of agreement to a certain statement, where such level of agreement is classified in a Likert scale (Likert, 1932). The response probability must be modeled as a function of the characteristics of the decision maker only. It is convenient to do so using an ordinal logit approach, since the responses to the indicators consist of a few integer values. We define the item response function $g$ relative to the answer of individual $n$ to indicator $I_{k}$ as:

$$
G_{I_{k}, n}^{s}=g\left(Z_{n} ; \alpha_{k}^{s}\right)+v_{k n}^{s}
$$

where $\alpha_{k}^{s}$ is a indicator- and class-specific vector of parameters to be estimated, $v_{k n}^{s} \sim \operatorname{Logistic}(0,1)$ is a disturbance term and $G_{I_{k}, n}^{s}$ is a latent continuous variable that increases with the level of agreement $\ell$ to indicator $k$. The probability of answering $\ell$ comes defined by:

$$
P_{n}\left(I_{k}=\ell \mid s\right)=P\left(\tau_{\ell-1}^{s}<G_{I_{k}=\ell, n}^{s} \leq \tau_{\ell}^{s}\right)
$$

where $\ell=1, \ldots, L$ is the level of agreement to indicator $I_{k}$ and $\tau_{\ell}^{s}$ are strictly increasing classspecific thresholds defining an ordinal relation between the utility $G_{I_{k}, n}^{s}$ and the answers to $I_{k}$. The probability of an individual $n$ providing an answer $\ell$ to indicator $I_{k}$ is:

$$
P_{n}\left(I_{k}=\ell \mid s\right)=\frac{1}{1+\exp \left(-\left(\tau_{\ell}^{s}-g\left(Z_{n} ; \alpha_{k}^{s}\right)\right)\right)}-\frac{1}{1+\exp \left(-\left(\tau_{\ell-1}^{s}-g\left(Z_{n} ; \alpha_{k}^{s}\right)\right)\right)}
$$

Because a complete set of thresholds $\tau_{\ell}$, for $\ell=1, \ldots, L-1$, cannot be fully identified, it is necessary to set the scale parameter of the logistically-distributed error term to 1 and to fix one 
of the thresholds (Greene and Hensher, 2009). For example the first threshold can be fixed to zero $\left(\tau_{1}^{s}=0\right)$, then only the difference between thresholds $\left(\delta_{\ell}\right)$ has to be estimated given that $\tau_{\ell}^{s}=\tau_{\ell-1}^{s}+\delta_{\ell-1}^{s}$ for $\ell=2, \ldots, L-1$.

The parameters of the joint model of choice, class-membership and response to psychometric indicators can be simultaneously estimated by maximizing the following likelihood function:

$$
\mathscr{L}=\prod_{n}\left\{\sum_{s}\left\{P_{n}(i \mid s) \prod_{k} P_{n}\left(I_{k} \mid s\right)\right\} P_{n}(s)\right\},
$$

where we adopt the following simplified notations:

$$
\begin{gathered}
P_{n}(i \mid s):=\prod_{i} P_{n}(i \mid s)^{y_{i n}} \\
P_{n}\left(I_{k} \mid s\right):=\prod_{\ell} P_{n}\left(I_{k}=\ell \mid s\right)^{y_{k \ell n}}
\end{gathered}
$$

where $y_{i n}$ is a variable that assumes the value of 1 if individual $n$ chose alternative $i$ and 0 otherwise, and $y_{k \ell n}$ assumes the value of 1 if individual $n$ chose answer $\ell$ to the indicator (or question) $I_{k}$.

If the probability of providing a certain answer to the indicator is modeled as a constant, the likelihood function then becomes

$$
\mathscr{L}=\prod_{n}\left\{\sum_{s}\left\{P_{n}(i \mid s) \prod_{k} \pi_{k s}\right\} P_{n}(s)\right\},
$$

where $P_{n}\left(I_{k} \mid s\right)$ is replaced by $\pi_{k s}$, which does not depend on attributes of the decision maker and can be estimated directly as a parameter.

The proposed approach, together with the basic latent class model and the constant-based indicator measurement model, is applied in two case studies of mode choice, presented next.

\section{Case study for the city of Nice}

We consider first a data set from a travel survey performed in 2008 for a region around the city of Nice, France (CERTU, 2008). The survey reports performed trips by purpose and mode (origin, destination and travel time) for a given day and socioeconomics of the travelers. Additionally, the survey requested respondents to provide their opinion on different statements related to their living environment and to different transport modes. The responses were rated on a three-point Likert 
scale, ranging to 1 for a disagreement response to 3 for an agreement response. Information for the non-chosen transport modes of each trip was collected using a script for automatic web-parsing that collected information from the official website of the public transport system and from GIS data of the road network. More details on the survey and data processing can be found in Nguyen (2012). Only morning trips to work including the additional opinion statements were considered for estimation, adding up to a total of 1687 trips.

\subsection{Model specification}

We follow the same exploratory approach described in Walker and Li (2007) for the definition of the class-specific choice model and the class-membership model specifications. This means that we define a generic specification for the utility of each transport mode alternative that does not vary across classes, except for the values of the class-specific parameters $\left(\beta^{s}\right)$. For the classmembership model we do not explore the optimal number of classes to consider, because it is out of the scope of this paper and because the use of two classes should be enough to test the method proposed here.

From the exploratory approach, we identified two classes, namely well-off suburban families (class 1) and eco-friendly city dwellers (class 2).

The class-membership functions depend on socioeconomic characteristics of the decision maker and its household. They are defined as follows:

$$
\begin{aligned}
f\left(Z_{n}, \gamma^{1}\right) & =\mathrm{ASC}^{1}+\gamma_{S P C}^{1} \mathrm{SPC}_{n}+\gamma_{\text {size }}^{1}{\operatorname{size} \_h h_{n}}_{n} \gamma_{\text {cars }}^{1} \operatorname{cars}_{n} \\
f\left(Z_{n}, \gamma^{2}\right) & =0
\end{aligned}
$$

where $\mathrm{ASC}^{1}$ is the class-specific constant for latent class 1 . We consider three main explanatory variables $\left(Z_{n}\right)$ : the size of the household $\left(\operatorname{size}_{-} h_{n}\right)$, the number of cars in the household $\left(\operatorname{cars}_{n}\right)$ and a dummy if the socio-professional category ${ }^{1}$ of the traveler $\left(\mathrm{SPC}_{n}\right)$ is $\mathrm{high}^{2}$, as a proxy of a high income level.

For the class-specific mode choice model we consider only two alternatives: Car (including both as driver and as passenger) and Public Transport (PT). Cost (C) and travel time (TT) are the main

\footnotetext{
${ }^{1}$ The socio-professional categories are a classification of job types according to required education level and expected income. They are defined, computed and provided by the National Institute of Statistics and Economic Studies of France (INSEE), http://www.insee.fr/en/.

${ }^{2}$ Corresponding to category 3 of the INSEE classification: Liberal, intellectual and managerial professions
} 
attributes of each alternative but additional information regarding the availability of car for each household and spatial attributes of the origin and destination zones of the trip is also available. The class-specific utility functions for each alternative are the following:

$$
\begin{aligned}
V\left(X_{C A R}, Z_{n}, \beta^{s}\right) & =\beta_{c o s t}^{s} \mathrm{C}_{C A R}+\beta_{T T}^{s} \mathrm{TT}_{C A R}+\beta_{c a r \_a v}^{s} \mathrm{cars}_{n}+\beta_{c \_O D}^{s}{\mathrm{c} \_O D_{n}}_{1}+\beta_{\text {park }}^{s} \mathrm{park}_{n} \\
V\left(X_{P T}, Z_{n}, \beta^{s}\right) & =\mathrm{ASC}_{P T}^{s}+\beta_{c o s t}^{s} \mathrm{C}_{P T}+\beta_{T T}^{s} \mathrm{TT}_{P T}+\beta_{P T_{-a v}}^{s} \mathrm{PT}_{-} \mathrm{O}_{n}+\beta_{c D}^{s} \mathrm{c} \_\mathrm{D}_{n}
\end{aligned}
$$

where $\mathrm{c}_{-} \mathrm{OD}_{n}$ is a dummy variable indicating that the trip performed by individual $n$ has both its origin and destination in the central zones of the area of study (urban center of Nice) while $c_{-} \mathrm{D}_{n}$ is a similar indicator but considering only a central destination of the trip. PT_O $\mathrm{O}_{n}$ is a measure of the density of public transport stops by square kilometer at the origin of the trip and park ${ }_{n}$ is a dummy variable indicating that individual $n$ has available parking at her destination.

Of all the opinions and statements included in the survey we consider the following two to be used as indicators:

- I1: We need to build more parking lots downtown.

- I2: The future of urban transportation for the central city is the bicycle.

We select these two indicators because they measure attitudes towards specific transport modes that are in the extremes of the transport mode spectrum (motorization and environmental-wise). While a positive answer to statement I1 indicates a desire for a more car-based city, agreement with statement I 2 indicates a desire for a "greener" city. While the relation of statement I1 with a preference for one of the alternatives (the car) is clear, statement I 2 is about a mode that is not considered among the alternatives of the mode choice problem. However, our hypothesis is that a positive perception of biking as an urban transport mode indicates a more human-centered vision of the city and this should have an influence on the choice between car and public transport. It is important to notice that both statements are about the central urban area; this means that agreement with both or any of them indicates concern for the city center, while indifference or disagreement probably indicates that the respondent is indifferent with what happens to the city center and probably develops her activities outside of it. Because the data contains very few neutral responses (less than 5\% for both indicators), we aggregate the responses in two levels: agreement and disagreement. We include the neutral responses in the disagreement level, since both are associated with a certain lack of interest.

We define the item response functions of each indicator as follows: 


$$
\begin{aligned}
& g\left(X_{1 n} ; \alpha_{1}^{s}\right)=\mathrm{ASC}_{I 1}^{s}+\alpha_{\text {cars }}^{s} \mathrm{cars}_{n} \\
& g\left(X_{2 n} ; \alpha_{2}^{s}\right)=\mathrm{ASC}_{I 2}^{s}+\alpha_{c_{-} O}^{s} \mathrm{c}_{-} \mathrm{O}_{n}
\end{aligned}
$$

We assume that the item response function of indicator I1 depends on the number of cars in the household of the respondent and, therefore, we expect a positive parameter for $\alpha_{\text {cars }}$ since a higher utility relative to the choice of the level of I1 will be associated with an agreement with the statement. Indicator I 2 depends on the location of the origin of the trip being inside of central Nice. Besides the obvious fact that the statement refers to the city center, the hypothesis is that central residents (all trips considered in estimation start from the residential location) are more likely to be bike users (or have a positive perception of these mode) because the city center concentrates more biking infrastructure than the surrounding suburbs and most bike trips are performed in the central region, where activity opportunities are located at reasonable distances for bicycle trips.

\subsection{Estimation results}

Three models are estimated for the Nice case study. First, we consider a standard latent class model (LCM1) that does not include indicators and therefore uses the probability expression given by equation (5) with the utility specifications defined by (14) and (15).

The second model (LCM2) is an extension of the first one but considers indicators, where the response probabilities $\pi_{k s}$ are estimated directly as parameters for each class and possible answer to the indicator. The likelihood function is given by equation (13).

Finally, the third model (LCM3) is also an extension of the first one but uses the method proposed in this paper to measure indicators and, therefore, the response probabilities are estimated using equation (9) with the utility specifications given by (16). The likelihood function is given by equation (10).

All models were estimated using Biogeme (Bierlaire, 2003; Bierlaire and Fetiarison, 2009). The estimation process considered an exploratory and incremental approach, beginning with the estimation of simpler models and specifications, in order to obtain good starting points (initial values of estimates) for the estimation of more complex models.

We remark that all three models have the same specification for the utility function relative to the choice model and for the class-membership function. Estimation results for all three models are shown in Table 1. 
Table 1: Estimation results - Case study for the city of Nice

\begin{tabular}{|c|c|c|c|c|c|c|c|}
\hline & & $\overline{\mathrm{LCl}}$ & & $\mathrm{LCl}$ & & $\mathrm{LC}$ & \\
\hline & parameter & estimate & t-test & estimate & t-test & estimate & t-test \\
\hline & $A S C_{P T}^{1}$ & 2.09 & 0.75 & 2.01 & 0.55 & 0.080 & 0.03 \\
\hline & $A S C_{P T}^{2}$ & -1.21 & -1.42 & -1.30 & -1.59 & -1.14 & $-2.08 * *$ \\
\hline & $\beta_{\text {cost }}^{1}$ & -0.499 & -1.41 & -0.413 & -0.81 & -0.458 & $-1.80 *$ \\
\hline & $\beta_{\text {cost }}^{2}$ & -1.81 & $-3.14 * *$ & -1.60 & $-3.26 * *$ & -1.42 & $-4.61 * *$ \\
\hline 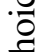 & $\beta_{T T}^{1}$ & -0.346 & $-2.03 * *$ & -0.324 & -1.59 & -0.213 & -1.07 \\
\hline 0 & $\beta_{T T}^{2}$ & -0.125 & $-2.98 * *$ & -0.115 & $-3.23 * *$ & -0.113 & $-4.24 * *$ \\
\hline ह & $\beta_{c a r_{-} a v}$ & 1.59 & 1.50 & 1.59 & 1.27 & 1.09 & 1.08 \\
\hline & $\beta_{c_{-} O D}$ & -1.22 & $-2.74 * *$ & -1.23 & $-2.81 * *$ & -1.17 & $-3.01 * *$ \\
\hline & $\beta_{P T \_a v}$ & 0.0115 & $1.76^{*}$ & 0.0113 & $1.84 *$ & 0.0108 & $1.95^{*}$ \\
\hline & $\beta_{c D}^{s}$ & 1.35 & $2.43 * *$ & 1.24 & $2.25 * *$ & 1.11 & $2.26^{* *}$ \\
\hline & $\beta_{\text {park }}$ & 2.82 & $5.26^{* *}$ & 2.77 & $5.31 * *$ & 2.64 & $5.52 * *$ \\
\hline & $A S C^{1}$ & -1.64 & -1.03 & -2.05 & -1.30 & -3.90 & -1.48 \\
\hline s & $\gamma_{S P C}^{1}$ & 1.00 & 1.45 & 1.39 & $1.97 * *$ & 1.80 & $1.97 * *$ \\
\hline$\ddot{U}$ & $\gamma_{\text {cars }}^{1}$ & 1.46 & 0.58 & 1.96 & 0.80 & 2.61 & 0.96 \\
\hline & $\gamma_{s i z e}^{1}$ & 2.55 & 1.28 & 2.17 & 0.98 & 5.26 & 1.36 \\
\hline & $P\left(I 1_{\text {agree }} \mid 1\right)$ & - & - & $0.80^{a}$ & $2.82 * *$ & - & - \\
\hline & $P\left(I 1_{\text {agree }} \mid 2\right)$ & - & - & $0.71^{a}$ & $7.11 * *$ & - & - \\
\hline$\ddot{E}$ & $P\left(I 2_{\text {agree }} \mid 1\right)$ & - & - & $0.55^{a}$ & $6.98 * *$ & - & - \\
\hline 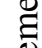 & $P\left(12_{\text {agree }} \mid 2\right)$ & - & - & $0.68^{a}$ & $6.30 * *$ & - & - \\
\hline 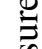 & $\mathrm{ASC}_{I 1}^{1}$ & - & - & - & - & 1.44 & $2.27 * *$ \\
\hline$\widetilde{\Xi}$ & $\alpha_{\text {cars }}^{1}$ & - & - & - & - & -0.0844 & -0.29 \\
\hline 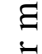 & $\mathrm{ASC}_{I 1}^{2}$ & - & - & - & - & 0.550 & $1.90 *$ \\
\hline 串 & $\alpha_{\text {cars }}^{2}$ & - & - & - & - & 0.314 & 1.46 \\
\hline שֶ: & $\mathrm{ASC}_{I 2}^{1}$ & - & - & - & - & 0.894 & $2.04 * *$ \\
\hline$\Xi$ & $\alpha_{c}^{1}$ & - & - & - & - & -1.11 & $1.80 *$ \\
\hline & $\mathrm{ASC}_{I 2}^{2}$ & - & - & - & - & 0.565 & $2.44 * *$ \\
\hline & $\alpha_{c_{-} O}^{2}$ & - & - & - & - & 0.189 & 0.61 \\
\hline & -like for choices & -144.10 & & -144.41 & & -145.31 & \\
\hline & -likelihood for I1 & - & & -371.20 & & -370.01 & \\
\hline & -likelihood for I2 & - & & -429.52 & & -426.31 & \\
\hline
\end{tabular}

${ }^{a}$ Only the probabilities for agreement answers are provided, the probabilities for disagreement answers can be computed as $1-P\left(I_{k} \mid s\right)$. 
For all three models, the parameters for the mode choice model have the expected sign and no change of sign is observed across models. It was noticed that the difference between classes was not significant for some parameters and they were merged in a single parameter. This is the case for all parameters in the choice model, with the exception of the constants, the cost and travel time parameters . Class 1 is more sensitive to travel time than class 2 while class 2 perceives a higher dis-utility for the cost than class 1 . Both classes have a higher probability of choosing car if it is available and the probability of choosing public transport increases with the presence of stops near of the residential location. If the trip starts or ends in the central city, the probability of choosing car diminishes while the opposite happens for public transport. Availability of parking space at the destination makes the car more attractive for both classes.

The signs of parameters in the class-membership model help to characterize the classes. In all three models, the probability of belonging to class 1 increases with the socio-professional category, the number of cars and the size of the household. This means that class 1 probably corresponds to members of high income, large families that have a tendency to use the car. This is also consistent with the observed higher sensitive to time and lower sensitive to cost observed for class 1. However, the basic model (LCM1) does not have any significant parameter in the class-membership model, while LCM2 and LCM3 have significant parameters only for the socioprofessional category. This result indicates that no conclusion about class membership should be drawn from LCM1 but, simultaneously, it suggests that using psychometric data helps to better identify classes.

In terms of indicator measurement, the estimated probabilities of model LCM2 are consistent with the shares by type of answer observed in the data. It is possible to see that class 1 tends to give more agreement answers to the parking-related question (I1) while, at the same time, tends to give fewer agreement answers to the bicycle-related question (I2). This reinforces the idea that class 1 corresponds to car-using families of high income.

For model LCM3, since the indicators have only two possible levels of agreement, there is only one threshold, which is arbitrarily set to zero without loss of generality (Greene and Hensher, 2009). This means that, for both indicators, a positive utility triggers an agreement response while a negative utility triggers a disagreement response. Results for this model indicate that class 1 will almost systematically provide an agreement answer to indicator I1, regardless of the number of cars (because $\alpha_{\text {cars }}^{1}$ is of low magnitude and extremely not significant) while class 2 also tends to give agreement answers but increasing with the number of cars. Answers to question I2 also tend to be of agreement for class 1 , except for when they are dwellers of the central city. Class 2 
individuals tend to increase their level of agreement to I 2 if they are located in central Nice. This result provides some behavioral insight on the perceptions and attitudes of each class, indicating that class 1 is more irrestrictively biased towards the car and likes the idea of more bicycles as long as they are not disturbed by them. Class 2 seems to be more consistent, providing more importance to parking if they are car users and having a positive bias towards the bicycle, especially if they are potential users. It is important to notice that, when computing the shares by answer for each indicator of LCM3 using equation (9), they have very similar values to those observed in LCM2 and the data.

In the case of models integrating several components, like latent class choice models, likelihood values cannot be compared directly. It is possible, however, to compute likelihood values for each measurement component of the model directly as the log of the sum of the probabilities of the observed choices/answers to psychometric indicators. These likelihood values are shown at the bottom of Table 1 . All three models perform similarly in terms of fit to observed choices, with LCM3 having a slightly lower fit. The fit for the indicator-measurement model is similar between LCM2 and LCM3 which suggests that LCM2 should be kept since it has fewer parameters. However, the additional parameters in LCM3 play the role of providing additional behavioral insight into each class, something that cannot be done through constants alone (as it is the case of LCM2). In terms of predicting capabilities, all models perform similarly, as it can be seen in Table 2, where estimated market shares of car and PT by class and overall classes are shown. All models tend to underestimate the share of car, which was $83 \%$ in the original data. However, in terms of estimation of value of time (VOT), LCM1 and LCM2 lead to a rather high VOT for class 1 while LCM3 provides a value of 27 Euros/hour, which is closer to the reference value of 10 Euros/hour for the year $2001^{3}$ (CGP, 2001). This may be caused by the lack of significance of the cost parameter in LCM1 and LCM2, which renders the VOT for these two models less reliable.

The application of the proposed methodology on the data set from the Nice works as a meaningful proof of concept. However, despite the fact that extensive specification testing was performed on the Nice data set, many parameters show low statistical significance for all models. This motivated the application of the methodology on a more reliable data set coming from a different case study, presented in the next section.

\footnotetext{
${ }^{3}$ Using the official annual interest rates for France this value is 13,3 Euros/hour in 2008. Source: http://epp.eurostat.ec.europa.eu/portal/page/portal/interest_rates/data/main_tables
} 
Table 2: Market shares and value of time - Nice case study

\begin{tabular}{|ll|r|r|r|}
\cline { 3 - 5 } \multicolumn{1}{l|}{} & Car share [\%] & PT share [\%] & VOT [Euro/hour] \\
\hline \multirow{2}{*}{ LCM1 } & Class 1 & 87.09 & 12.91 & 41.60 \\
& Class 2 & 73.96 & 26.04 & 4.14 \\
& Overall & 80.60 & 19.40 & 21.89 \\
\hline \multirow{2}{*}{ LCM2 } & Class 1 & 87.11 & 12.89 & 47.07 \\
& Class 2 & 75.20 & 24.80 & 4.31 \\
& Overall & 80.49 & 19.51 & 21.53 \\
\hline \multirow{2}{*}{ LCM33 } & Class 1 & 88.25 & 11.75 & 27.90 \\
& Class 2 & 75.95 & 24.05 & 4.77 \\
& Overall & 80.57 & 19.43 & 12.43 \\
\hline
\end{tabular}

\section{Switzerland case study}

Data from a revealed preferences travel survey conducted in 2009 in rural areas of Switzerland was collected (EPFL, 2011). The travel survey describes socioeconomics and the complete tour of trips of the respondent for a given weekday including mode, purpose, departure and arrival times. Additionally, as psychometric indicators, the survey collected responses in terms of level of agreement to a series of statements about the environment, the transport system, lifestyle preferences and mobility habits (for more details see () Hurtubia et al., 2010). The answers were collected using a five point Likert scale ranging from strong disagreement (level 1) to a strong agreement (level 5). After data cleaning and processing, the observations of trips and set of answers to the psychometric indicators of 1763 respondents were considered for estimation. In total, 2265 trips with an associated choice of transport mode were recorded, given that a respondent could report several trips per day.

\subsection{Specification}

This case study is an extension of the model and results presented by Atasoy et al. (2013). For comparison purposes, the specification of utility functions, definition of latent classes and selection of psychometric indicators are the same as those proposed in the aforementioned article.

Atasoy et al. (2013) identified class 1 as individuals living with their families who have high income while class 2 corresponds to single individuals who live alone or with their parents.

The class-membership functions are the following

$$
\begin{aligned}
& f\left(Z_{n}, \gamma^{1}\right)=\operatorname{ASC}^{1}+\gamma_{\text {child }}^{1} \text { child }_{n}+\gamma_{\text {inc }}^{1} \text { high_inc }_{n} \\
& f\left(Z_{n}, \gamma^{2}\right)=\gamma_{\text {single }^{2}} \text { single }_{n}
\end{aligned}
$$


Table 3: Mode choice model specification - Swiss case study

\begin{tabular}{|c|c|c|c|c|c|}
\hline & \multicolumn{3}{|c|}{ Variable (Class 1) } & \multicolumn{2}{|c|}{ Variable (Class 2) } \\
\hline Parameter & $\overline{V_{P M M}}$ & $\overline{V_{P T}}$ & $V_{S M}$ & $V_{P M M}$ & $\overline{V_{P T}}$ \\
\hline$A S C_{P M M}^{1}$ & 1 & - & - & - & - \\
\hline$A S C_{P M M}^{2}$ & - & - & - & 1 & - \\
\hline$A S C_{S M}^{1}$ & - & - & 1 & - & - \\
\hline$\beta_{\text {cost }}^{1}$ & Cost $_{P M M}$ & $\operatorname{Cost}_{P T}$ & - & - & - \\
\hline$\beta_{\text {cost }}^{2}$ & - & - & - & Cost $_{P M M}$ & $\operatorname{Cost}_{P T}$ \\
\hline$\beta_{T T, P M M}^{1}$ & $T T_{P M M}$ & - & - & - & - \\
\hline$\beta_{T T, P M M}^{2}$ & - & - & - & $T T_{P M M}$ & - \\
\hline$\beta_{T T, P T}^{1}$ & - & $T T_{P T}$ & - & - & - \\
\hline$\beta_{T T, P T}^{2}$ & - & - & - & - & $T T_{P T}$ \\
\hline$\beta_{\text {distance }}^{1}$ & - & - & Dist $_{S M}$ & - & - \\
\hline$\beta_{\text {cars }}$ & cars & - & - & cars & - \\
\hline$\beta_{\text {children }}^{1}$ & children & - & - & - & - \\
\hline$\beta_{\text {children }}^{2}$ & - & - & - & children & - \\
\hline$\beta_{\text {language }}$ & French & - & - & French & - \\
\hline$\beta_{\text {work }}^{1}$ & WorkTrip & - & - & - & - \\
\hline$\beta_{\text {work }}^{2}$ & - & - & - & WorkTrip & - \\
\hline$\beta_{\text {urban }}$ & - & Urban & - & - & Urban \\
\hline$\beta_{\text {student }}$ & - & Student & - & - & Student \\
\hline$\beta_{\text {bikes }}^{1}$ & - & - & bikes & - & - \\
\hline
\end{tabular}

The class-membership model depends on three main socioeconomic attributes of the decision maker: a dummy variable indicating if the traveler $n$ belongs to a household with children $\left(\operatorname{child}_{n}\right)$, a dummy indicating if the income in the household is above CHF 8000 per month (high_inc $c_{n}$ ) and a dummy indicating if individual $n$ lives alone or with his parents $\left(\right.$ single $\left._{n}\right)$.

The mode choice model considers three alternatives: Private Motorized Modes (PMM), including car as driver, car as passenger, motorcycle and taxi, Public Transport (PT), including bus, metro and train, and Soft Modes (SM) including bicycle and walking. The class-specific utilities for mode choice are described in each column of Table 3. Because there was no observations of soft modes chosen by individuals falling in the "single" category, this alternative was made unavailable for class 2. This modeling assumption implies that conditional on the fact that an individual is in class 2 , he does not have any access to bike or walk.

In Table 3, $T T_{P M M}$ and $T T_{P T}$ are the travel times for private modes and public transport respectively, cars is the number of cars in the household, children is the number of children under age 15 in the household and bikes is the number of bicycles available to the members of the household. French is a dummy variable indicating if the respondent lives in the French part of Switzerland, WorkTrip is a dummy indicating that the purpose of the trip was work, Urban is a dummy indi- 
cating the origin or destination of the trips is in an urban area and Student is a dummy indicating if the respondent is a student (up to the university or trainee level).

After a factor analysis process, Atasoy et al. (2013) selected the following statement of the survey to be used as indicators:

- I1 (PT and children): It is hard to take public transport when I travel with my children.

- I2 (Flexibility of car): With my car, I can go where I want whenever I want.

- I3 (Family oriented): I would like to spend more time with my family and friends.

The item response functions of each indicator are the following.

$$
\begin{aligned}
& g\left(X_{1 n} ; \alpha_{1}^{S}\right)=A S C_{I 1}^{S}+\alpha_{\text {Children }^{s} \text { HasChildren }_{n}} \\
& g\left(X_{2 n} ; \alpha_{2}^{s}\right)=A S C_{I 2}^{s}+\alpha_{\text {cars }}^{s} \text { cars }_{n} \\
& g\left(X_{3 n} ; \alpha_{3}^{S}\right)=A S C_{I 3}^{S}+\alpha_{\text {family }}^{s} \text { HasChildren }_{n} \text { working }_{n}
\end{aligned}
$$

The answer to indicator I1 will be affected by a dummy indicating the presence of children in the household; the number of cars in the household affects the answer to question I 2 and the answer to indicator I3 depends on the interaction of two dummy variables indicating that the person has children and a full time job.

\subsection{Estimation results}

As in the case study of Section 4, three models were estimated for the Swiss case. The first one (LCM1) is simply an integrated choice and latent class model without indicators. The second one (LCM2) incorporates indicators and estimates the item response probabilities directly as parameters, using the likelihood function given by (13). The third one (LCM3) uses the methodology proposed in this paper and is estimated by maximizing the likelihood function given by (10).

All models were estimated using Biogeme (Bierlaire, 2003; Bierlaire and Fetiarison, 2009) following the same exploratory and incremental approach described in Section 4.2.

As for the Nice case study, all models have the same specification for the utility functions relative to the choice model and the class-membership function. Results for the choice model and the 
Table 4: Estimation results - Swiss case study

\begin{tabular}{|c|c|c|c|c|c|c|c|}
\hline & & \multicolumn{2}{|c|}{ LCM 1} & \multicolumn{2}{|c|}{ LCM2 } & \multicolumn{2}{|c|}{ LCM3 } \\
\hline & Parameters & estimate & t-test & estimate & t-test & estimate & t-test \\
\hline \multirow{19}{*}{$\begin{array}{l}.00 \\
\frac{0}{0} \\
\frac{d}{0} \\
\frac{0}{0} \\
0 \\
\Sigma\end{array}$} & $A S C_{P M M}^{1}$ & -0.417 & -0.417 & -0.945 & $-3.83 * *$ & -1.25 & $-4.30 * *$ \\
\hline & $A S C_{P M M}^{2}$ & -0.571 & -1.49 & -0.936 & $-3.37 * *$ & -0.731 & $-2.54 * *$ \\
\hline & $A S C_{S M}^{1}$ & 0.587 & $1.67 *$ & 0.512 & $1.70^{*}$ & 0.642 & $2.07 * *$ \\
\hline & $\beta_{\text {cost }}^{1}$ & -4.15 & $-2.12 * *$ & -2.70 & $-3.14 * *$ & -1.23 & -1.53 \\
\hline & $\beta_{\text {cost }}^{2}$ & -30.5 & $-4.83 * *$ & -30.2 & $2.82 * *$ & -39.1 & $-6.98 * *$ \\
\hline & $\beta_{T T, P M M}^{1}$ & -0.211 & -0.42 & -1.61 & $-4.77 * *$ & -1.30 & $-3.80 * *$ \\
\hline & $\beta_{T T, P M M}^{2}$ & -26.8 & $-4.96 * *$ & -11.1 & $-6.83 * *$ & -10.6 & $-6.46 * *$ \\
\hline & $\beta_{T T, P T}^{1}$ & -0.257 & -0.98 & -0.692 & $-3.62 * *$ & -0.701 & $-3.55 * *$ \\
\hline & $\beta_{T T, P T}^{2}$ & -8.91 & $-4.85^{* *}$ & -4.45 & $-5.90 * *$ & -3.91 & $-5.35 * *$ \\
\hline & $\beta_{\text {distance }}^{1}$ & -18.4 & $-8.42 * *$ & -19.9 & $-9.54 * *$ & -19.8 & $-9.10 * *$ \\
\hline & $\beta_{\text {cars }}$ & 1.24 & $10.18 * *$ & 1.23 & $11.34 * *$ & 1.29 & $11.18 * *$ \\
\hline & $\beta_{\text {children }}^{1}$ & 0.403 & $2.76^{* *}$ & 0.404 & $4.83 * *$ & 0.346 & $3.47 * *$ \\
\hline & $\beta_{\text {children }}^{2}$ & -0.434 & $-1.89 *$ & -1.03 & $-1.72 *$ & 0.211 & 0.97 \\
\hline & $\beta_{\text {language }}$ & 1.20 & $5.71 * *$ & 1.20 & $6.79 * *$ & 1.20 & $6.22 * *$ \\
\hline & $\beta_{\text {work }}^{1}$ & -0.990 & $-3.98 * *$ & -0.785 & $-4.85 * *$ & -0.623 & $-3.37 * *$ \\
\hline & $\beta_{\text {work }}^{2}$ & 0.0881 & 0.22 & -0.130 & -0.43 & -0.396 & -1.34 \\
\hline & $\beta_{\text {urban }}$ & 0.528 & $3.20 * *$ & 0.390 & $2.82 * *$ & 0.459 & $3.23 * *$ \\
\hline & $\beta_{\text {student }}$ & 3.73 & $8.37 * *$ & 3.70 & $8.45 * *$ & 3.95 & $8.86^{* *}$ \\
\hline & $\beta_{\text {bikes }}^{1}$ & 0.400 & $4.96 * *$ & 0.205 & $3.21 * *$ & 0.214 & $3.26 * *$ \\
\hline \multirow{4}{*}{$\begin{array}{l}\text { 岕 } \\
\text { J }\end{array}$} & $A S C_{\text {class }}$ & -0.215 & -0.86 & -0.629 & $-3.25 * *$ & -0.589 & $-3.39 * *$ \\
\hline & $\gamma_{\text {child }}^{1}$ & 0.136 & 0.51 & 3.92 & $4.84 * *$ & 0.967 & $5.41 * *$ \\
\hline & $\gamma_{i n c}^{1}$ & 0.693 & $2.76^{* *}$ & 0.460 & $2.22 * *$ & 0.684 & $4.50 * *$ \\
\hline & $\gamma_{\text {single }}^{2}$ & 0.408 & 1.34 & 0.704 & $3.57 * *$ & 0.743 & $3.33 * *$ \\
\hline \multirow{4}{*}{\multicolumn{2}{|c|}{$\begin{array}{l}\text { Log-like for choices } \\
\text { Log-likelihood for I1 } \\
\text { Log-likelihood for I2 } \\
\text { Log-likelihood for I3 }\end{array}$}} & -994.7 & & -1032.5 & & -1006.7 & \\
\hline & & - & & -2068.4 & & -2033.1 & \\
\hline & & - & & -2202.6 & & -2151.5 & \\
\hline & & - & & -2160.6 & & -2153.5 & \\
\hline
\end{tabular}

class-membership model are shown in Table 4. The estimated item response probabilities for LCM2 and the parameters for the indicator measurements of LCM3 are shown in Tables 6 and 7 of the Appendix respectively.

The choice model parameters for cost and time show the expected sign for all classes in the three models. Most of the remaining parameters show intuitive values and no change of sign across models, with some exceptions that are not relevant due to the low significance of the estimates.

The estimates for the class-membership model confirm that class 1 corresponds to high income individuals living with their family while class 2 corresponds to single individuals with lower income. In general the inclusion of indicators (in both LCM2 and LCM3) allows for the estimation of more significant parameters in the class-membership model. 
Regarding the measurement of indicators, both LCM2 and LCM3 generate response probabilities (see Table 6) that are consistent with observed response rates. Some additional behavioral interpretation is possible when looking at the indicator measurement parameters of LCM3 (see Table 7 in the appendix). For example, for indicator I1 (difficulty of using public transport with children), it is possible to see that class 2 has a strong inertial tendency to be indifferent, confirming that individuals in class 2 are likely to have no children. On the other hand, individuals in class 1 show a more heterogeneous behavior in their responses, which tends to be of disagreement when the household has children.

In terms of fit to observed choices, the basic model (LCM1) has a better fit than LCM2 and LCM3, which is to be expected given the more complex likelihood functions of the models including indicators. The model proposed in this paper (LCM3) has a better fit than the one using only constants to model the answers to indicators (LCM2) both in terms of fit to observed choices and to responses to psychometric indicators.

The models forecast market shares with some differences, as seen in Table 5. These market shares were computed using weights (for a detailed explanation see Atasoy et al. (2013)) and therefore are comparable to actual market shares of Private Motorized Modes (66\%), Public Transport (28\%) and Soft Modes (6\%). In terms of value of time LCM1 predicts a counter-intuitive higher value of time for class 2. The models including indicators (LCM2 and LCM3) produce a more intuitive VOT for each class, although LCM3 predicts a much higher VOT for private motorized modes (PMM) in class 1. The reference VOT for Switzerland is $27.66 \mathrm{CHF} /$ hour for business travels by car (Axhausen et al., 2008). However, estimation data was obtained from a survey that was conducted in rural areas of Switzerland, where income tends to be higher, while the reference VOT considers both rural and urban areas. This, besides the fact that many individuals in class 1 have at least a wage of $50 \mathrm{CHF} /$ hour $^{4}$, justifies considering the results provided by LCM3 as reasonable since, under some circumstances, the value of travel time savings should be close to the wage level (Jara-Diaz, 2007). This, however, requires further research to reach a conclusion.

\section{Conclusions}

We propose a new type of model specification that incorporates psychometric indicators into integrated choice and latent class models through an ordinal logit model. Moreover the ordinal logit model relates the answers to the indicators with socioeconomic characteristics of the respondents, hence allowing for a better characterization of the latent classes.

\footnotetext{
${ }^{4}$ computed as CHF 8000 divided by 160 hours of work per month
} 
Table 5: Market shares and value of time - Swiss case study

\begin{tabular}{|c|c|c|c|c|c|c|}
\hline \multicolumn{2}{|c|}{ Models } & PMM [\%] & PT [\%] & SM [\%] & VOT PMM [CHF/h] & VOT PT [CHF/h] \\
\hline \multirow{3}{*}{ LCM1 } & Class 1 & 60.97 & 28.73 & 10.30 & 3.06 & 3.72 \\
\hline & Class 2 & 60.41 & 39.59 & - & 52.63 & 17.53 \\
\hline & Overall & 61.23 & 33.81 & 4.96 & 28.97 & 10.94 \\
\hline \multirow{3}{*}{ LCM2 } & Class 1 & 54.91 & 36.13 & 8.96 & 35.78 & 15.38 \\
\hline & Class 2 & 65.73 & 34.27 & - & 22.05 & 8.84 \\
\hline & Overall & 62.7 & 32.35 & 4.94 & 29.53 & 12.40 \\
\hline \multirow{3}{*}{ LCM3 } & Class 1 & 51.79 & 38.01 & 10.2 & 63.27 & 16.21 \\
\hline & Class 2 & 70.98 & 29.02 & - & 34.16 & 5.99 \\
\hline & Overall & 61.74 & 33.69 & 4.57 & 36.94 & 18.40 \\
\hline
\end{tabular}

The method is tested in two mode choice case studies for the region of Nice, France, and rural areas of Switzerland. Results show that the inclusion of the ordinal measurement of psychometric indicators generates significantly different estimates for the class-membership model. The additional behavioral insights provided by the parameters of the indicator-measurement equations allows for a richer analysis of the latent classes, giving the analyst more tools to identify different market segments.

The proposed method forecasts values of time of different magnitudes when compared with latent class models that estimate the item response probabilities of the indicators as single parameters. In the Nice case study, the method proposed in this paper produced values that were clearly closer to the reference ones. In the Swiss case study our method predict much higher values of time for the high income class.

One of the advantages of the proposed methodology is the closed form of the ordinal logit used for measurement of the indicators. This allows for a simpler estimation procedure, without the need of integration techniques as it is in most cases when latent variables are included in choice models.

Some of the estimates in the models presented here have a low significance level and some of the utility functions for classes and indicators have considerably simple specifications. This is due to the complexity of the models and the relatively scarce number of observations available for each case study. We believe that using a larger set of observations should allow to incorporate more explanatory variables in the class-membership and indicator measurement utilities, therefore expanding the possibilities of behavioral analysis and market segmentation. 


\section{References}

Atasoy, B., Glerum, A. and Bierlaire, M. (2013). Attitudes towards mode choice in Switzerland, disP - The Planning Review 49(2): 101 -117.

Axhausen, K. W., Hess, S., Koenig, A., Abay, G., Bates, J. J. and Bierlaire, M. (2008). Income and distance elasticities of values of travel time savings: New Swiss results, Transport Policy 15(3): 173-185.

Ben-Akiva, M. E. and Boccara, B. (1995). Discrete choice models with latent choice sets, International Journal of Research in Marketing 12(1): 9-24.

Ben-Akiva, M. E. and Lerman, S. R. (1985). Discrete Choice Analysis: Theory and Application to Travel Demand, MIT Press, Cambridge, Ma.

Ben-Akiva, M., McFadden, D., Train, K., Walker, J., Bhat, C., Bierlaire, M., Bolduc, D., BörschSupan, A., Brownstone, D., Bunch, D. et al. (2002). Hybrid choice models: Progress and challenges, Marketing Letters 13(3): 163-175.

Ben-Akiva, M., Walker, J., Bernardino, A., Gopinath, D., Morikawa, T. and Polydoropoulou, A. (2002). Integration of choice and latent variable models, In perpetual motion: Travel behaviour research opportunities and application challenges, Elsevier, Amsterdam pp. 431470.

Bhat, C. (1997). An endogenous segmentation mode choice model with an application to intercity travel, Transportation Science 31(1): 34-48.

Bierlaire, M. (2003). Biogeme: a free package for the estimation of discrete choice models, Proceedings of the Swiss Transport Research Conference, Ascona, Switzerland.

Bierlaire, M. and Fetiarison, M. (2009). Estimation of discrete choice models: extending biogeme, Proceedings of the 9th Swiss Transport Research Conference, Ascona, Switzerland.

Boxall, P. and Adamowicz, W. (2002). Understanding heterogeneous preferences in random utility models: A latent class approach, Environmental \& Resource Economics 23(4): 421-446.

CERTU (2008). Enquête ménage déplacements des alpes-maritimes, Technical report, Centre d'Etudes sur les Réseaux, les Transports, l'Urbanisme et les constructions publiques, France. URL: $h t t p: / / w w w . c e r t u . f r$ 
CGP (2001). Transports: choix des investissements et coût des nuisances, La documentation française.

Collins, L. M. and Lanza, S. T. (2010). Latent class and latent transition analysis: with application in the social, behavioral, and health sciences, Wiley Series in Probability and Statistics.

EPFL (2011). Projet de recherche sur la mobilité combinée, OPTIMA: Rapport définitif de l'enquête de préférences révélées, Technical report, CarPostal Suisse SA and Transport and Mobility Laboratory, Ecole Polytechnique Fédérale de Lausanne.

URL: http://infoscience.epfl.ch/record/167785

Glerum, A. and Bierlaire, M. (2012). The use of word data to measure perception in hybrid choice models, Proceedings of the 13th International Conference on Travel Behavior Research, Toronto, Canada.

Gopinath, D. A. (1995). Modeling heterogeneity in discrete choice processes: application to travel demand, $\mathrm{PhD}$ thesis, Massachusetts Institute of Technology.

Greene, W. H. and Hensher, D. A. (2009). Modeling ordered choices, Cambridge University Press.

Greene, W. and Hensher, D. (2003). A latent class model for discrete choice analysis: contrasts with mixed logit, Transportation Research Part B: Methodological 37(8): 681-698.

Hess, S., Ben-Akiva, M., Gopinath, D. and Walker, J. (2011). Advantages of latent class over continuous mixture of logit models, Technical report, Working paper, University Press, Harrisburg.

Hess, S., Shires, J. and Jopson, A. (2013). Accommodating underlying pro-environmental attitudes in a rail travel context: Application of a latent variable latent class specification, Transportation Research Part D: Transport and Environment 25(0): 42 - 48.

Hosoda, T. (1999). Incorporating unobservable heterogeneity in discrete choice model: mode choice model for shopping trips, $\mathrm{PhD}$ thesis, Massachusetts Institute of Technology.

Hurtubia, R., Atasoy, B., Glerum, A., Curchod, A. and Bierlaire, M. (2010). Considering latent attitudes in mode choice: The case of Switzerland, Proceedings of the World Conference on Transport Research, Lisbon, Portugal.

Jara-Diaz, S. (2007). Transport economic theory, Elsevier Science. 
Kamakura, W. A. and Russell, G. J. (1989). A probabilistic choice model for market segmentation and elasticity structure, Journal of Marketing Research 26(4): pp. 379-390.

Keane, M. and Wasi, N. (2012). Comparing alternative models of heterogeneity in consumer choice behavior, Journal of Applied Econometrics .

Koutsopoulos, H. and Farah, H. (2012). Latent class model for car following behavior, Transportation Research Part B: Methodological 46(5): 563-578.

Lazarsfeld, P. and Henry, N. (1968). Latent structure analysis, Houghton, Mifflin.

Likert, R. (1932). A technique for the measurement of attitudes, Archives of Psychology 22(140).

McFadden, D. (1986). The choice theory approach to market research, Marketing Science 5(4): 275-297.

McFadden, D. and Train, K. (2000). Mixed MNL models for discrete response, Journal of applied Econometrics 15(5): 447-470.

Morey, E., Thacher, J. and Breffle, W. (2006). Using angler characteristics and attitudinal data to identify environmental preference classes: A latent-class model, Environmental and Resource Economics 34: 91-115.

Nguyen, M. H. (2012). Mode choice models for the city of nice using psychometric indicators and latent variables, Master's thesis, Ecole Polytechnique Fédérale de Lausanne, Switzerland.

Shen, J. (2009). Latent class model or mixed logit model? a comparison by transport mode choice data, Applied Economics 41(22): 2915-2924.

Walker, J. and Ben-Akiva, M. (2002). Generalized random utility model, Mathematical Social Sciences 43(3): 303 - 343.

Walker, J. L. and Li, J. (2007). Latent Lifestyle Preferences and Household Location Decisions, Journal of Geographical Sytems 9: 77-101.

Wen, C.-H., Wang, W.-C. and Fu, C. (2012). Latent class nested logit model for analyzing highspeed rail access mode choice, Transportation Research Part E: Logistics and Transportation Review 48(2): 545 - 554.

Wen, C. and Lai, S. (2010). Latent class models of international air carrier choice, Transportation Research Part E: Logistics and Transportation Review 46(2): 211-221. 
Zhang, J., Kuwano, M., Lee, B. and Fujiwara, A. (2009). Modeling household discrete choice behavior incorporating heterogeneous group decision-making mechanisms, Transportation Research Part B: Methodological 43(2): 230-250. 


\section{Appendix}

Table 6: Item response probabilities for LCM2 - Swiss case study

\begin{tabular}{|l|lc|lc|}
\cline { 2 - 6 } \multicolumn{1}{c|}{} & \multicolumn{2}{c|}{$s=1$} & \multicolumn{2}{c|}{$s=2$} \\
\hline Probability & estimate & t-test & estimate & $\mathrm{t}$-test \\
\hline$P(I 1=1 \mid s)$ & 0.166 & $13.00^{* *}$ & 0.002 & 0.78 \\
$P(I 1=2 \mid s)$ & 0.246 & $16.14^{* *}$ & 0.008 & 0.67 \\
$P(I 1=3 \mid s)$ & 0.306 & $14.11^{* *}$ & 0.958 & $34.60^{* *}$ \\
$P(I 1=4 \mid s)$ & 0.176 & $13.45^{* *}$ & 0.029 & 2.36 \\
$P(I 1=5 \mid s)$ & 0.106 & $a$ & 0.003 & $a$ \\
\hline$P(I 2=1 \mid s)$ & 0.031 & $5.60^{* *}$ & 0.020 & $3.31^{* *}$ \\
$P(I 2=2 \mid s)$ & 0.033 & $5.73^{* *}$ & 0.027 & $3.94^{* *}$ \\
$P(I 2=3 \mid s)$ & 0.121 & $11.10^{* *}$ & 0.169 & $10.80^{* *}$ \\
$P(I 2=4 \mid s)$ & 0.371 & $23.87^{* *}$ & 0.364 & $18.03^{* *}$ \\
$P(I 2=5 \mid s)$ & 0.444 & $a$ & 0.420 & $a$ \\
\hline$P(I 3=1 \mid s)$ & 0.013 & $3.63^{* *}$ & 0.004 & 1.35 \\
$P(I 3=2 \mid s)$ & 0.047 & $6.84^{* *}$ & 0.040 & $4.80^{* *}$ \\
$P(I 3=3 \mid s)$ & 0.254 & $17.08^{* *}$ & 0.414 & $19.78^{* *}$ \\
$P(I 3=4 \mid s)$ & 0.491 & $29.91^{* *}$ & 0.430 & $20.46^{* *}$ \\
$P(I 3=5 \mid s)$ & 0.195 & $a$ & 0.112 & $a$ \\
\hline
\end{tabular}

Table 7: Indicator measurement parameters for LCM3 - Swiss case study

\begin{tabular}{|c|cr|lr|}
\cline { 2 - 5 } \multicolumn{1}{c|}{} & \multicolumn{2}{c|}{$s=1$} & \multicolumn{2}{c|}{$s=2$} \\
\hline parameter & estimate & \multicolumn{1}{c|}{$\mathrm{t}$-test } & estimate & \multicolumn{1}{c|}{$\mathrm{t}$-test } \\
\hline$A S C_{I 1}^{s}$ & 2.04 & $12.97^{* *}$ & 5.18 & $3.13^{* *}$ \\
$\alpha_{\text {Children }}^{s}$ & -1.28 & $-6.85^{* *}$ & 3.87 & $10.50^{* *}$ \\
$\delta_{1, I 1}^{s}$ & 1.57 & $15.02^{* *}$ & 0.461 & 0.35 \\
$\delta_{2, I 1}^{s}$ & 1.96 & $10.77^{* *}$ & 7.40 & $4.08^{* *}$ \\
$\delta_{3, I 1}^{s}$ & 1.18 & $6.66^{* *}$ & 1.94 & $9.12^{* *}$ \\
\hline$A S C_{I 2}^{s}$ & 2.26 & $8.60^{* *}$ & 3.31 & $9.81^{* *}$ \\
$\alpha_{\text {cars }}^{s}$ & 5.11 & $7.03^{* *}$ & 2.84 & $4.29^{* *}$ \\
$\delta_{1, I 2}^{s}$ & 0.845 & $5.15^{* *}$ & 0.781 & $3.51^{* *}$ \\
$\delta_{2, I 2}^{s}$ & 1.32 & $9.82^{* *}$ & 1.32 & $9.82^{* *}$ \\
$\delta_{3, I 2}^{s}$ & 1.79 & $17.44^{* *}$ & 1.74 & $17.06^{* *}$ \\
\hline$A S C_{I 3}^{s}$ & 3.86 & $12.88^{* *}$ & 6.26 & $3.20^{* *}$ \\
$\alpha_{\text {family }}^{s}$ & 0.309 & $2.05^{* *}$ & 0.987 & $5.76^{* *}$ \\
$\delta_{1, I 3}^{s}$ & 1.31 & $5.26^{* *}$ & 3.33 & $1.76^{*}$ \\
$\delta_{2, I 3}^{s}$ & 2.07 & $13.51^{* *}$ & 2.69 & $13.96^{* *}$ \\
$\delta_{3, I 3}^{s}$ & 2.39 & $19.99^{* *}$ & 2.08 & $18.84^{* *}$ \\
\hline
\end{tabular}

*Parameter significant with $90 \%$ confidence.

**Parameter significant with $95 \%$ confidence.

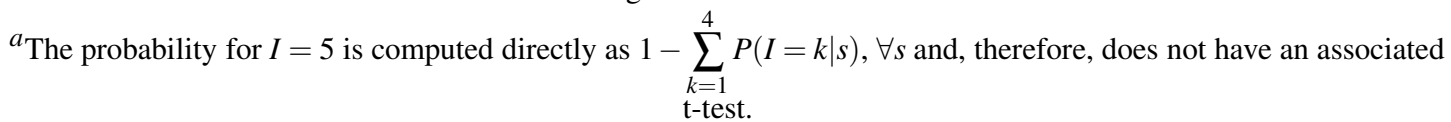

